\title{
Mistreatment during Facility Based Child-Birth Care in a Nigerian Tertiary Hospital
}

\section{Ohaeri Beatrice M*, Onyeneho Chiemerigo A and Suliyat 0}

Department of Nursing, Faculty of Clinical Sciences, College of Medicine, University of Ibadan, Nigeria

*Corresponding author: Beatrice M Ohaeri, Department of Nursing, Faculty of Clinical Sciences, College of Medicine, University of Ibadan, Nigeria, Tel: +2348161352904; Email: bmkohaeri@

Research Article

Volume 4 Issue 4

Received Date: June 29, 2020

Published Date: July 30, 2020

DOI: $10.23880 /$ nhij- 16000226 yahoo.co.uk

\section{Abstract}

Background: Globally, thousands of women die annually from complications during pregnancy or child-birth especially in developing countries. The experience of mistreatment by women during childbirth has been identified as a barrier to utilization of facility based childbirth care globally, which could increase maternal mortality and morbidity. Hence, this study assessed the prevalence and forms of mistreatment experienced during facility -based childbirth care by women, in a Nigerian hospital. Method: A cross-sectional descriptive research design was used and simple random sampling technique was used to select calculated sample of 72 women who consented, from postnatal clinic of University College Hospital. A validated structured interviewer-administered questionnaire adapted from Community Survey Tool, was used to collect data on prevalence and forms of mistreatment during delivery among the women. Data were analyzed using descriptive statistics of mean and ChiSquare at $5 \%$ level of significance. Ethical approval was obtained.

Result: The Mean age of the women is $32 \pm 2.1$ years. All of the women were married (100\%), and (97.2\%) had tertiary education. Prevalence of mistreatment was $16.7 \%$ with verbal abuse (shouting, 15.3\%), physical abuse (slapping the thigh, $1.4 \%$ ), while stigma and discrimination were $1.4 \%$. On maternal intervention, almost half (43.1\%) had Caesarean section done. Majority (70.8\%) had vaginal examinations, $67 \%$ reported that their experience of vaginal examination was uncomfortable. Conclusion: The findings revealed that women experience mistreatment during delivery. Hence, there is need for education of health care workers on the implication of mistreatment and also the need to provide support to women during delivery, to enhance their childbirth experience. This could promote the utilization of facility-based child care given by skilled birth attendant and ultimately reduce maternal and perinatal mortality and morbidity rate.

Keywords: Mistreatment; Facility; Child-birth; Care

\section{Introduction}

Globally, thousands of women die annually from complications during pregnancy, child birth or post-partum period with most deaths occurring in developing countries [1]. Despite the substantial improvement in utilization of Skill Birth Attendant, Unicef reports that facility based deliveries remains low in several regions like sub-Saharan
Africa, South Asia, Middle East and North Africa as well as in developing countries [2]. One of the efforts made globally in reducing maternal and perinatal mortality and morbidity is by increasing the rates of facility based childbirth [3] as well as improve the quality of care that women experience during delivery [4]. One of the areas of promoting quality care is to avoid mistreatment during delivery. Women who receive childbirth services have reported various experiences with 
reference to mistreatment during labour and delivery.

Mistreatment during childbirth is the violation of rights and trust between women and their health care providers [5]. Mistreatment ranges from physical abuse, verbal abuse, stigma and discrimination, failure to meet professional standard of care, poor rapport between women and providers and health system constraints [6]. WHO stated that one-third of women experienced mistreatment during childbirth in health care facilities [7]. In Nigeria the rate of mistreatment is reported to be higher [8]. WHO states that "every woman has the right to the highest attainable standard of health, which includes the right to dignified, respectful health care" [5]. However, studies have shown that health care providers who are meant to provide dignified care mistreat women during delivery period like stigmatizing and discriminating women based on socio-demographic characteristics such as age, ethnicity, level of education, marital status and in some cases HIV status and the failure to meet professional standard of care such as little or no explanation before procedures like vaginal examination and episiotomy which are often common low quality care practices taking place in health facilities. Also, rapport between women and care provider is often low or non-existent in some cases thereby diminishing women's trust in the health system as well as disrespectful, undignified care and abusive care during childbirth from health providers [9-13]. These tend to thwart the rights of these women as well as quality of care thereby serving as disincentive for women to seek facility care [14]. This can further lead to maternal and newborn mortality and morbidity which could have been averted if these women utilized the services of skilled birth attendants.

The various stages or time in which one or more forms of mistreatments are experienced by women in labour have been reported by several studies ranges between 30 minutes before birth until 15 mins after birth $[7,14]$. Due to these various forms of mistreatment experienced by women during childbirth, most women have demonstrated lack of motivation to seek skilled birth attendant care and have resorted in seeking care outside of the health care facilities from unskilled birth attendant whom they believe will not mistreat them [12-14]. Also such mistreatment may also have effects on future maternal health-seeking behavior [14]. Furthermore, apart from the fact that women will end up seeking birth services outside of health facilities, mistreatment can have other negative health implications on women which could be immediate or after delivery [15-17].

Discouraging mistreatment of women by health care workers and encouraging women to speak out when mistreated will help to promote high quality care and motivate women to seek skilled birth attendant in health care facilities thereby promoting positive care experiences for women and improve maternal and newborn health [17]. Hence, this study aims at assessing mistreatment during childbirth in health care facilities.

\section{Materials and Methods}

\section{Study Design}

A cross-sectional descriptive research design was used.

\section{Study Setting}

Postnatal clinic of University College Hospital, Ibadan, Nigeria.

\section{Study Population}

Postnatal mothers who received labour/delivery services at the UCH presenting for their postnatal visit 6 weeks post-delivery.

\section{Sampling Technique and Sample Size}

A simple random sampling technique by balloting (yes/ no) and those who picked yes were recruited for the study. A sample size of 72 women which was calculated using Yamane 1967 formula, from postnatal clinic of University College Hospital were recruited for the study.

\section{Instrument for Data Collection}

An adapted validated structured questionnaire named Community Survey Tool by Bohren et al, 2018 which collects data on the socio-demographic information, obstetric history, birth experiences (including mistreatment, vaginal examination, companionship and pain relief), child birth outcomes, interventions, postpartum depression, future child bearing intentions and satisfaction with care was used for the study. However, based on the study objectives the instrument was adapted to collect data only on prevalence of mistreatment, birth experiences like various forms of mistreatment (physical, verbal, stigma and discrimination), and patient's experience with vaginal examination and response to maternal interventions amongst these women. A total of 72 questionnaires that were completely filled were used for data analysis.

Data analysis: Data collected on the objectives were analyzed using descriptive statistics of frequencies, percentages, mean and standard deviation. Inferential statistics of Chi-square test at 5\% level of significance was used to ascertain the association between parity and mistreatment among these women.

Ethical approval: Ethical approval was obtained from UI/ UCH ethical review committee. Ethical principles were observed. 


\section{Results}

Table 1 show that the mean age $\pm S$.D of the women is
$32 \pm 2.1,97.2 \%$ has tertiary education, $79.2 \%$ are Yoruba, $66.7 \%$ are Christians, $87.5 \%$ had one to two living children, and $47.2 \%$ had one to two previous births.

\begin{tabular}{|c|c|c|c|}
\hline Variable & Response & Frequency & Percentage (\%) \\
\hline \multicolumn{4}{|c|}{ Age group (years) } \\
\hline $20-24$ & & 3 & 4.2 \\
\hline $25-29$ & & 10 & 13.9 \\
\hline $30-34$ & & 40 & 55.6 \\
\hline Above 34 & & 19 & 26.4 \\
\hline Mean Age $32 \pm 2.1$ & & & \\
\hline \multicolumn{4}{|c|}{ Ethnic group } \\
\hline Hausa & & 3 & 4.2 \\
\hline Igbo & & 9 & 12.5 \\
\hline Yoruba & & 57 & 79.2 \\
\hline Tiv & & 3 & 4.2 \\
\hline \multicolumn{4}{|l|}{ Religion } \\
\hline Islam & & 23 & 31.9 \\
\hline Christianity & & 48 & 66.7 \\
\hline Traditional & & 1 & 1.4 \\
\hline \multicolumn{4}{|c|}{ Occupation } \\
\hline Artisan & & 9 & 12.5 \\
\hline Business & & 28 & 38.9 \\
\hline Civil service & & 18 & 25 \\
\hline Student & & 12 & 16.7 \\
\hline Private company & & 5 & 6.9 \\
\hline \multicolumn{4}{|l|}{ Marital status } \\
\hline Married & & 72 & 100 \\
\hline \multicolumn{4}{|c|}{ Highest level of education } \\
\hline Secondary & & 1 & 2.8 \\
\hline Tertiary & & 71 & 97.2 \\
\hline \multicolumn{4}{|l|}{ Number of pregnancy(ies) } \\
\hline One & & 25 & 34.7 \\
\hline Two & & 23 & 31.9 \\
\hline Three & & 11 & 15.3 \\
\hline Four & & 9 & 12.5 \\
\hline Above four & & 3 & 4.2 \\
\hline No response & & 1 & 1.4 \\
\hline \multicolumn{4}{|c|}{ Number of previous birth } \\
\hline None & & 33 & 45.8 \\
\hline One & & 18 & 25 \\
\hline Two & & 16 & 22.2 \\
\hline Three & & 5 & 5.9 \\
\hline
\end{tabular}




\section{Nursing \& Healthcare International Journal}

\begin{tabular}{|c|c|c|}
\hline \multicolumn{3}{|c|}{ Number of living child(ren) } \\
\hline One & 41 & 56.9 \\
\hline Two & 22 & 30.6 \\
\hline Three & 6 & 8.3 \\
\hline Four & 3 & 4.2 \\
\hline \multicolumn{3}{|c|}{ Mode of birth for most recent pregnancy } \\
\hline Vagina & 42 & 58.3 \\
\hline Caesarean birth & 30 & 41.7 \\
\hline \multicolumn{3}{|c|}{ Number of babies at most recent birth } \\
\hline Singleton & 70 & 97.2 \\
\hline Twins & 2 & 2.8 \\
\hline \multicolumn{3}{|c|}{ Sex of baby(ies) } \\
\hline Male & 40 & 55.6 \\
\hline Female & 32 & 44.4 \\
\hline
\end{tabular}

Table 1: Socio-demographic Characteristics of Respondents.

\section{Prevalence of Mistreatment Experienced By Women}

The result on the prevalence of mistreatment experienced by the women showed that only $16.7 \%$ of the respondents experienced one or other forms of mistreatment during childbirth in the hospital while $83.3 \%$ didn't experience any form of mistreatment.

Table 2 below reveals that only one (1.4\%) of the women experienced slap as a form of physical mistreatment during childbirth.

\begin{tabular}{|c|c|c|}
\hline \multirow{2}{*}{ Item } & \multicolumn{2}{|c|}{ Frequency (\%) } \\
\hline & No & Yes \\
\hline 1. Pinched by a health worker or other staff & $72(100.0)$ & $0(0.0)$ \\
\hline 2. Slapped by a health worker or other staff & $71(98.6)$ & $1(1.4)$ \\
\hline 3. Kicked by a health worker or other staff & $72(100.0)$ & $0(0.0)$ \\
\hline 4. Punched by a health worker or other staff & $72(100.0)$ & $0(0.0)$ \\
\hline 5. Hit with an instrument by a health worker or other staff & $72(100.0)$ & $0(0.0)$ \\
\hline $\begin{array}{l}\text { 6. Gagged (eg: something put across or in your mouth to prevent you from speaking or } \\
\text { making noise) by a health worker or other staff }\end{array}$ & $72(100.0)$ & $0(0.0)$ \\
\hline 7. Physically tied to the bed by a health worker or other staff & $72(100.0)$ & $0(0.0)$ \\
\hline 8. Held down to the bed forcefully by a health worker or other staff & $72(100.0)$ & $0(0.0)$ \\
\hline $\begin{array}{l}\text { 9. Forceful downward pressure placed on your abdomen before the baby came out (fundal } \\
\text { pressure) }\end{array}$ & $72(100.0)$ & $0(0.0)$ \\
\hline 10. Had another form of physical force used against you (please specify) & $72(100.0)$ & $0(0.0)$ \\
\hline
\end{tabular}

Table 2: Physical form of abuse or mistreatment.

At any time during the birth of your last child, were you:

Table 3 below reveals that $15.3 \%$ of the respondents were shouted or screamed at by a health worker or other staff, $11.1 \%$ were threatened verbally with" if you don't comply, you and your baby will have poor outcome", $4.2 \%$ were insulted, $5.6 \%$ were scolded at, $2.8 \%$ were threatened with medical procedure, $1.4 \%$ were threatened with physical violence and verbally abused.

During labour and birth of your child, were you: 


\begin{tabular}{|l|c|c|}
\hline \multicolumn{1}{|c|}{ Item } & \multicolumn{2}{c|}{ Frequency (\%) } \\
\cline { 2 - 3 } & No & Yes \\
\hline 1. Shouted or screamed at by a health worker or other staff & $61(84.7)$ & $11(15.3)$ \\
\hline 2. Insulted by a health worker or other staff & $69(95.8)$ & $3(4.2)$ \\
\hline 3. Scolded by a health worker or other staff & $68(94.4)$ & $4(5.6)$ \\
\hline 4. Mocked by a health worker or other staff & $72(100.0)$ & $0(0.0)$ \\
\hline $\begin{array}{l}\text { 5. The health worker or other staff member made negative comments about your physical } \\
\text { appearance (such as your weight, private parts, cleanliness or other parts of your body)? }\end{array}$ & $72(100.0)$ & $0(0.0)$ \\
\hline $\begin{array}{l}\text { 6. The health worker or other staff member made negative comments about your baby's physical } \\
\text { appearance (such as his/her appearance, sex or other aspects of the baby)? }\end{array}$ & $72(100.0)$ & $0(0.0)$ \\
\hline $\begin{array}{l}\text { 7. The health worker or other staff member made negative comments to you regarding your sexual } \\
\text { activity? }\end{array}$ & $72(100.0)$ & $0(0.0)$ \\
\hline $\begin{array}{l}\text { 8. The health worker or other staff threatened you with a medical procedure (such as episiotomy } \\
\text { or caesarean section)? }\end{array}$ & $70(97.2)$ & $2(2.8)$ \\
\hline 9. The health worker or other staff threatened you with physical violence? & $71(98.6)$ & $1(1.4)$ \\
\hline $\begin{array}{l}\text { 10. The health worker or other staff threatened that if you did not comply, you or your baby would } \\
\text { have poor outcome }\end{array}$ & $64(88.9)$ & $8(11.1)$ \\
\hline $\begin{array}{l}\text { 11. The health worker or other staff threatened to withhold care from you or your baby } \\
\text { 12. The health worker or other staff blamed you for something that happened to you or your baby } \\
\text { during your time in hospital }\end{array}$ & $72(100.0)$ & $0(0.0)$ \\
\hline 13. The health worker or other staff hissed at you during your time in hospital? & $72(100.0)$ & $0(0.0)$ \\
\hline 14. Other use of verbal abuse against you (please specify) & $71(98.6)$ & $0(0.0)$ \\
\hline
\end{tabular}

Table 3: Verbal form of Abuse or mistreatment.

Table 4 below reveals that only one (1.4\%) of the respondents was discriminated with comments regarding ethnicity, race, tribe or culture.

During the time in hospital, the following events occur

\begin{tabular}{|l|c|c|}
\hline \multicolumn{1}{|c|}{ Item } & \multicolumn{2}{c|}{ Frequency (\%) } \\
\cline { 2 - 3 } & \multicolumn{1}{|c|}{ No } & Yes \\
\hline $\begin{array}{l}\text { 1. A health worker or staff made negative comments to you regarding your ethnicity, race, tribe } \\
\text { or culture: }\end{array}$ & $71(98.6)$ & $1(1.4)$ \\
\hline 2. A health worker or staff made negative comments to you regarding your religion: & $72(100.0)$ & $0(0.0)$ \\
\hline 3. A health worker or staff made negative comments to you regarding your age: & $72(100.0)$ & $0(0.0)$ \\
\hline $\begin{array}{l}\text { 4. A health worker or staff made negative comments to you regarding whether you were married } \\
\text { or not }\end{array}$ & $72(100.0)$ & $0(0.0)$ \\
\hline $\begin{array}{l}\text { 5. A health worker or staff made negative comments to you regarding your level of education or } \\
\text { literacy }\end{array}$ & $72(100.0)$ & $0(0.0)$ \\
\hline 6.A health worker or staff made negative comments to you regardingyour economic circumstances & $72(100.0)$ & $0(0.0)$ \\
\hline 7. A health worker or staff made negative comments to you regarding your HIV status & $72(100.0)$ & $0(0.0)$ \\
\hline
\end{tabular}

Table 4: Responses to Stigma and Discrimination.

\section{Result on Responses on Maternal Intervention}

The result on maternal intervention showed that $43.1 \%$ received caesarean section while $56.9 \%$ did not. Among those that had caesarean section, $41.7 \%$ reported that the procedure was explained to them, and they all agreed to the procedure before it was done. However, $68.1 \%$ said they didn't receive episiotomy while $31.9 \%$ received episiotomy, 
$23.6 \%$ of the respondents said the procedure was not explained to them before episiotomy was given.

\section{Result on Vaginal Examination Experience}

The result on experience with vaginal examination showed that majority $(70.8 \%)$ received vaginal examination while $29.2 \%$ did not. However, $67 \%$ of the respondents reported that their experience of vaginal examination was uncomfortable. Also, many (55.6\%) had vaginal examination explained to them why it was needed, $56.9 \%$ reported that the health worker asked for their permission before performing vaginal examination, and $59.7 \%$ had privacy secured before vaginal examination could be done on them.

Table 5 below reveals that there is significant $(\mathrm{p}=0.037)$ association between parity and experience of mistreatment.

\begin{tabular}{|c|c|c|c|c|c|c|c|}
\hline \multirow{2}{*}{\multicolumn{2}{|c|}{ No }} & \multicolumn{2}{|c|}{ Mistreatment } & \multirow{2}{*}{ d.f } & \multirow{2}{*}{$X^{2}$-value } & \multirow{2}{*}{ p-value } & \multirow{2}{*}{ Remark } \\
\hline & & Yes & & & & & \\
\hline \multirow{4}{*}{ Number of previous birth } & One & $18(25.4 \%)$ & $0(0.0 \%)$ & & & & \multirow{4}{*}{ Significant } \\
\hline & Two & $13(18.3 \%)$ & $3(4.2 \%)$ & & & & \\
\hline & Three & $5(7.0 \%)$ & $0(0.0 \%)$ & 3 & 7.31 & 0.037 & \\
\hline & None & $23(32.4 \%)$ & $9(12.7 \%)$ & & & & \\
\hline
\end{tabular}

Table 5: Cross - tabulation of parity and mistreatment.

\section{Discussion}

Findings from the study revealed a low prevalence of mistreatment. Similar studies have reported low prevalence of mistreatment during delivery among women $[6,18]$. The low prevalence of mistreatment can be attributed to the fact that most women believe the health workers were rebuking them for their good, to avoid giving birth to a dead baby due to their inability to cooperate and push their babies. It can also be seen in this study that most of the women have had more than one previous birth and has gone through the experience of childbirth. So they would believe that whatever action that was taken against them was for their own good since they ended up giving birth to a live baby despite being mistreated. Similar studies have reported that women believe that all actions by health care providers were for their own good even if they were hit or slapped $[12,15,16]$. Furthermore, being a tertiary and training institution for health professionals, it is imperative to say that the low prevalence can be associated with the fact that they could have been taught about mistreatment of women as well as its implication on the health of the women. An observational study could further be done to actually ascertain the existence of mistreatment during delivery since the findings of this study is based on the responses from the women and there could be bias in the findings. However, similar studies have reported a high prevalence of mistreatment during ante-natal, intra-natal and post-natal period $[8,12]$.

Various forms of mistreatment have been reported in this study. However, the most prevalent reported by these women were verbal abuse, not obtaining consent on episiotomy and vaginal examination from these women by health care providers, lack of privacy during vaginal examination. Similar studies have also shown verbal abuse to be a form of mistreatment experienced by women during delivery [6,18-20]. Some studies have reported that health care professionals do not obtain consent before carrying out vaginal examination, episiotomy as well as providing privacy of their client during vaginal examination [21,22]. Informed consent is one of the ethics guiding the practice of all health care professionals. Hence, it is important to provide all women with adequate information and obtain an informed consent prior to any procedure or invasive procedure such as vaginal examination and episiotomy. This is supported by World Health Organization's report [23]. Health care professionals should be educated or a continuing education programme on mistreatment can be enacted by health care institutions to further enhance the knowledge of health workers on the implication of mistreatment on the health of women and the impact it has in future childbirth especially in seeking facility based health care.

Furthermore, parity has shown to be associated with mistreatment in our study. This can also be seen as reported by some studies, which showed that women are treated differently based on their age, parity and socio-economic status [6,24]. This is significant as health workers might have the notion that women who have given birth to more than one child should have had exposure to child birth and would have known what the birthing process is like. So not getting appropriate cooperation from the women could trigger them into being unhappy with the woman's behavior thereby leading to mistreatment like verbal abuse.

\section{Recommendations}

Based on the findings from this study, we therefore 
recommend the following:

1. Health care workers especially doctors and midwives should be educated on the implications of mistreatment on the women and the impact on seeking facility based care services in the future by these women.

2. There is also need for health workers to sensitize the women on what to expect during labour, in terms of labour pain, importance of bearing down at the second stage of labour amongst others in-order to gain their cooperation during the delivery period.

3. There is need to sensitize women and other family members about right to respectful care and empowering them to report and challenge disrespectful and abusive practices.

4. Policies should be enacted by hospital management on obtaining informed consent before any procedure especially vaginal examination and episiotomy and measures to ensure it is implemented by health workers should be done.

5. The need to carry out an observational study on mistreatment of women in this health care institution as there could have been bias in the report of these women.

\section{Conclusion}

The findings from this study have shown that women experience mistreatment during delivery. Hence, there is need for education of health care workers on mistreatment and its implication on the health of women and also the need to provide support to women during delivery which will enhance their childbirth experience thereby promoting the utilization of facility based child care by skilled birth attendant care. This will lead to reduction in maternal and perinatal mortality and morbidity rate. There is also, need to provide support, resources and supervise the activities of health workers during delivery in the labour wards of all health care institutions as this will help to curb mistreatment during delivery. There is need to incorporate and evaluate evidenced based interventions like feedbacks from the women in-order to reduce the prevalence of mistreatment in healthcare facilities. Inform consent should be sort by health care professionals while carrying out any form of maternal intervention like episiotomy, caesarean section, vaginal examination on the women during delivery and privacy should be maintained.

\section{References}

1. UN (2000) World Health Organization and World Bank partner to combat maternal mortality. The World Bank group news release incidence of maternal mortality, United Nations.

2. Abid A, Anna G, Celine L, Ticiana M, UNICEF, et al. (2014) The State of the World's Children 2014 in Numbers:
Every Child Counts, pp: 116.

3. Vogel JP, Bohren MA, Tunçalp Ö, Oladapo OT, Adanu RM, et al. (2015) How women are treated during facility-based childbirth: Development and validation of measurement tools in four countries-Phase 1 formative research study protocol. Reproductive Health 12: 60.

4. Powell-Jackson T, Mazumdar S, Mills A (2015) Finacial incentives in health: new evidence from india's janani Suraksha Yojana. Journal of Health Econs 43: 154-169.

5. WHO (2015) The prevention and elimination of disrespect and abuse during facility-based childbirth. WHO statement 2014. Human production programme, World Health Organization, pp: 4.

6. Okafor II, Ugwu EO, Obi SN (2014) Disrespect and abuse during facility-based childbirth in a low-income country International Journal of Gynecology and Obstetrics 128(2): 110-113.

7. Ogunlaja, OA, Fehintola OA, Ogunlaja IP, Popoola G, Idowu A, et al. (2017) Respectful maternity care or Disrespect and abuse during maternity care; experience of pregnant women in Ogbomoso, South West Nigeria. Rwanda Medical Journal 74(3): 6-9.

8. WHO (2020) New WHO evidence on mistreatment of women during childbirth. Sexual and Reproductive Health, World Health Organization.

9. Bohren MA, Vogel JP, Hunter EC, Lutsiv O, Makh SK, et al. (2015). The Mistreatment of Women during Childbirth in Health Facilities Globally: A Mixed Methods Systematic Review. PloS One 12(6): e1001847.

10. Bohren MA, Mehrtash H, Fawole B, Maung TM, Balde MD, et al. (2019) How women are treated during facilitybased childbirth in four countries: a cross-sectional study with labour observations and community-based surveys 6736(19): 1-14.

11. Sando D, Kendall T, Lyatuu G, Ratcliffe H, McDonald K, et al. (2014) Disrespect and abuse during childbirth in Tanzania: Are women living with HIV more vulnerable? Journal of Acquired Immune Deficiency Syndromes 67(4): 228-234.

12. Nawab T, Erum U, Amir A, Khalique N, Ansari MA, Chauhan A (2019) Disrespect and abuse during facility based childbirth and its sociodemographic determinants - A barrier to healthcare utilization in rural population. Journal of Family Medicine And Primary Care 8(1): 239245.

13. Balde MD, Diallo BA, Bangoura A, Sall O, Soumah AM, et al. 
(2017) Perceptions and experiences of the mistreatment of women during childbirth in health facilities in Guinea : a qualitative study with women and service providers. Reproductive Health 14(1): 3.

14. Maya ET, Adu-Bonsaffoh K, Dako-Gyeke P, Badzi C, Vogel, JP, et al. (2018). Women's perspective of mistreatment during childbirth at health facilities in Ghana: findings from a qualitative study. Reproductive Health Matters 26(53):70-87.

15. Bohren MA, Vogel JP, Tunçalp Ö, Fawole B, Titiloye MA, et al. (2017) Mistreatment of women during childbirth in Abuja, Nigeria: A qualitative study on perceptions and experiences of women and healthcare providers. Reproductive Health 14(1): 9.

16. Orpin J, Puthussery S, Davidson R, Burden B. (2018). Women's experiences of disrespect and abuse in maternity care facilities in BenueState, Nigeria. BMC Pregnancy and Childbirth 181: 1-9.

17. Shroll AM, Kjaergaard H, Midtgaard J (2013) Encountering abuse in health care; lifetime experiences in postnatal women: a qualitative study. BMC pregnancy and childbirth 13: 74 .

18. Freedman LP, Kruk ME (2014) Disrespect and abuse of women in childbirth: Challenging the global quality and accountability agendas. The Lancet 384(9948): E42-E44.
19. Sudhinaraset M, Trelleaven E, Melo J, Singh K, Diamondsmith N (2016) Women's status and experiences of mistreatment during childbirth in Uttar Pradesh: a Mixed method study using cultural health capital theory. BMC pregnancy childbirth 16(1): 332.

20. Ijadunola MY, Olotu EA, Oyedun O0, Eferakeya SO, Ilesanmi FI, et al. (2019) Lifting the veil on disrespect and abuse in facility-based child birth care: Findings from South West Nigeria. BMC Pregnancy and Childbirth 19(1): 1-8.

21. WHO (1996) Care in normal Birth: a practical guide. Geneva: World Health Organization.

22. Sharma G, Penn-Kekana L, Halder K, Filipi V (2019) An investigation into mistreatment of women during labour and childbirth in maternity care facilities in Uttar Pradesh, India: a mixed method study. Reproductive Health 16(1): 7.

23. Hameed W, Avan BI (2018) Wonen's experience of mistreatment during childbirth: A comparative view of home and facility-based births in pakistan. PloS One 13(3): e0194601.

24. Bowser D, Hill K (2010) Exploring Evidence for Disrespect and abuse in facility- based childbirth: report of a landscape analysis USAID Tration Project: Harvard School of Public Health University research Co., LLC, pp: 1-57.

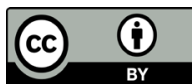

